\title{
Investigation on Flip Chip Solder Joint Fatigue With Cure-Dependent Underfill Properties
}

\author{
D. G. Yang, G. Q. Zhang, Leo J. Ernst, Cornelis van't Hof, J. F. J. M. Caers, H. J. L. Bressers, and J. H. J. Janssen
}

\begin{abstract}
A cure-dependent viscoelastic constitutive relation is applied to describe the curing process of epoxy underfill in flip chip on board (FCOB). The chemical shrinkage of the epoxy underfill during the curing process is applied via incremental initial strains. Thus, the stress and strain build-up, caused by the simultaneous increase in stiffness and shrinkage during the curing process, are simulated.

Accelerated fatigue experiments with thermal cycles from $-55{ }^{\circ} \mathrm{C}$ to $80{ }^{\circ} \mathrm{C}$ are carried out for a specially designed flip chip configuration. Based on the obtained curing induced initial stress and strain fields, thermo-mechanical predictions are presented for the test carriers. The solder bumps are modeled with temperature dependent visco-plastic properties. A combination of a Coffin-Manson based fatigue relation and a creep fatigue model is used as fatigue failure criterion. The results show that the finite element method (FEM)-based fatigue life predictions match better with the experimental results, if the curing induced initial stress state is taken into account. The effect of cure-induced hydrostatic stress is qualitatively investigated by using a modified energy partitioning damage model with a correction factor in the creep damage formulation to take into account the effect of the hydrostatic stress.
\end{abstract}

Index Terms-Curing process, flip chip, hydrostatic stress, solder fatigue, thermal cycling, underfill.

\section{INTRODUCTION}

$\mathbf{O}$ $\mathrm{NE}$ of the major reliability concerns with flip chips is the thermal fatigue of the solder bumps caused by mismatch in thermal expansion between the silicon die and the substrate. A polymer underfill is usually applied to improve the reliability of the package, by reducing the stresses of the solder bumps. As one of the additional-effects, however, the curing process of the underfill polymer will induce residual stress and strain fields. For simplicity reasons, in thermo-mechanical analyzes, the curing induced stress state is usually neglected by assuming a so-called "stress-free" temperature. Sometimes, this simplification can be partly justified by the fact that part of the initial stress will relax because of the material's viscoelastic behavior. However, in order to be able to conduct reliable curing process optimization and to predict thermo-mechanical reliability more accurately, it is vital to know the effect of the curing process dependent initial stresses and material properties.

Manuscript received February 1, 2003; revised March 3, 2003. This work was recommended for publication by Associate Editor r. S.-W. Lee upon evaluation of the reviewers' comments.

D. G. Yang, L. J. Ernst, and C. van't Hof are with the Delft University of Technology, Delft 2600 GA, The Netherlands (e-mail: 1.j.ernst@wbmt.tudelft.nl).

G. Q. Zhang, J. F. J. M. Caers, H. J. L. Bressers, and J. H. J. Janssen are with Philips CFT, Eindhoven 5600 MD, The Netherlands (e-mail: g.q.zhang@ philips.com).

Digital Object Identifier 10.1109/TCAPT.2003.815118
So far, many fatigue prediction models for solder joints have been proposed, which were reviewed recently in [1]. For the reliability of a flip chip on board (FCOB), much work has been done by many researchers to predict the solder fatigue, for example in [2]-[5]. Commonly, in using an assumed "stress-free" temperature, the cure-induced stresses due to chemical shrinkage are neglected, or a constant hydrostatic stress state in the solder bumps is assumed. To account for the effect of the hydrostatic stress on fatigue life prediction, Darbha et al. [5] modified an energy partitioning damage model by using a correction factor in the creep damage formulation. The underfill was considered to be elastic and the hydrostatic stress from the curing effect was obtained approximately by ramping temperature from the curing temperature to the glass transition temperature. It was concluded that the effect of the hydrostatic stress is significant. Nevertheless, so far, the effect of the curing process induced stress and strain on the solder fatigue life of FCOB has not been fully understood. The fatigue life of solder joints of FCOB appears to be dramatically influenced by the mechanical properties of underfill polymers, which are known to be strongly processing-dependent. Therefore, a reliable prediction of solder fatigue will depend on the ability to adequately model the thermo-mechanical properties of the constituents in the package. The process induced stress state and its influence should be well understood.

In order to investigate curing process induced stresses, a curedependent viscoelastic constitutive relation was originally proposed for polyester resins by Kiasat et al. [6], [7]. Ernst et al. [8] generalized the method. Further it was applied for describing cure-dependent viscoelastic behavior of epoxy resin [9], [10] and was used to investigate the effect of curing process induced stresses on the die cracking stress of a Flip Chip [11].

In this paper, the cure-dependent constitutive relation and the corresponding parameters for underfill epoxy are incorporated in the finite element analysis code MARC. Accelerated fatigue tests with thermal cycles from $-55^{\circ} \mathrm{C}$ to $80^{\circ} \mathrm{C}$ were performed for a specially designed flip chip configuration. Based on the obtained curing induced initial stress and strain fields, thermo-mechanical predictions are carried out for the test carriers. The solder bumps are modeled with temperature dependent visco-plastic properties. The cure-dependent viscoelastic model and a temperature dependent viscoelastic model are applied to describe the properties of the underfill resin during the curing process and subsequent thermal cycling, respectively. A combination of a Coffin-Manson based fatigue relation and a creep fatigue model is used to establish the combined fatigue damage. The effect of the cure-induced hydrostatic stress on the fatigue life prediction is discussed. The influence of the residual stresses induced in the curing process on the fatigue life of the solder bumps is investigated. 


\section{Modeling CuRE-DePENDENT Viscoelastic BehaVior OF UNDERFILL EPOXY}

During the curing process of thermosetting resins, a chemical cross-linking reaction takes place, accompanied with volumetric shrinkage and simultaneous stiffness build-up. When the shrinkage is (partly) constrained, as usually in the case of electronic packaging, shrinkage stress fields will be induced. In order to simulate the curing process, a three-dimensional cure-dependent constitutive relation for curing resin was developed and described by the following integral expression [6]-[9]:

$$
\begin{aligned}
S_{i}(t)=\int_{-\infty}^{t} & C_{i j}\{\alpha(\xi),(t-\xi)\} \\
& \times\left\{\left(\frac{\partial E_{j}}{\partial \xi}\right)_{\xi}-\left(\frac{\partial E_{j}^{*}}{\partial \xi}\right)_{\xi}\right\} d \xi .
\end{aligned}
$$

Here $S_{i}, E_{j}$ and $E_{j}^{*}$ are the components of the stress, strain and initial strain tensors, respectively. $C_{i j}$ represent the so-called cure-dependent relaxation modulus functions. $\alpha(\xi)$ represents the curing parameter, which is a function of the "load application time" (or curing time) $\xi$. Assuming isotropy, the relaxation modulus functions can be described by bulk and shear relaxation modulus functions as

$$
\begin{aligned}
C_{i j}\{\alpha(\xi),(t-\xi)\}=K\{\alpha(\xi) & ,(t-\xi)\} \cdot V_{i j} \\
& +G\{\alpha(\xi),(t-\xi)\} \cdot D_{i j} .
\end{aligned}
$$

Here $V_{i j}$ and $D_{i j}$ are the volumetric and deviatoric component matrices.

The generalized Maxwell model with a limited number of elements (Prony series) is used for approximating the shear and bulk relaxation moduli functions

$$
K\{\alpha(\xi),(t-\xi)\} \approx \sum_{n=1}^{N} K^{n}\{\alpha(\xi)\} \cdot e^{-(t-\xi) / \tau_{n}}
$$

and

$$
G\{\alpha(\xi),(t-\xi)\} \approx \sum_{n=1}^{N} G^{n}\{\alpha(\xi)\} \cdot e^{-(t-\xi) / \tau_{n}}
$$

where $G^{n}\{\alpha(\xi)\}$ and $K^{n}\{\alpha(\xi)\}$ are stiffness coefficients and $\tau_{n}$ are the relation times.

In order to implement the constitutive equation into a numerical simulation package, it needs to be written into an incremental form. The total curing time is subdivided into a number of subintervals $\left[t_{m-1}, t_{m}\right]$. Linear strain increments $\Delta E_{j}\left(t_{m}\right)$ are assumed over the time step $h=t_{m}-t_{m-1}$. Equation (1) can than be written as

$$
\begin{aligned}
& S_{i}\left(t_{m}\right)=e^{-h / \tau_{n}} \cdot S_{i}\left(t_{m-1}\right) \\
& \quad+C_{i j}\left\{\alpha\left(t_{m}\right)\right\} \cdot \Delta E_{j}\left(t_{m}\right) \cdot\left(\frac{\tau_{n}}{h}\right) \cdot\left(1-e^{-h / \tau_{n}}\right)
\end{aligned}
$$

with

$$
\begin{aligned}
\Delta S_{i}\left(t_{m}\right)=\left[\sum_{n=1}^{N} \frac{\tau_{n}}{h} \cdot\left(1-e^{-h / \tau_{n}}\right) \cdot C_{i j}\left\{\alpha\left(t_{m}\right)\right\}\right] & \times\left[\Delta E_{j}\left(t_{m}\right)-\Delta E_{j}^{*}\left(t_{m}\right)\right] \\
& +\sum_{n=1}^{N}\left(e^{-h / \tau_{n}}-1\right) \cdot S_{i}\left(t_{m-1}\right) .
\end{aligned}
$$

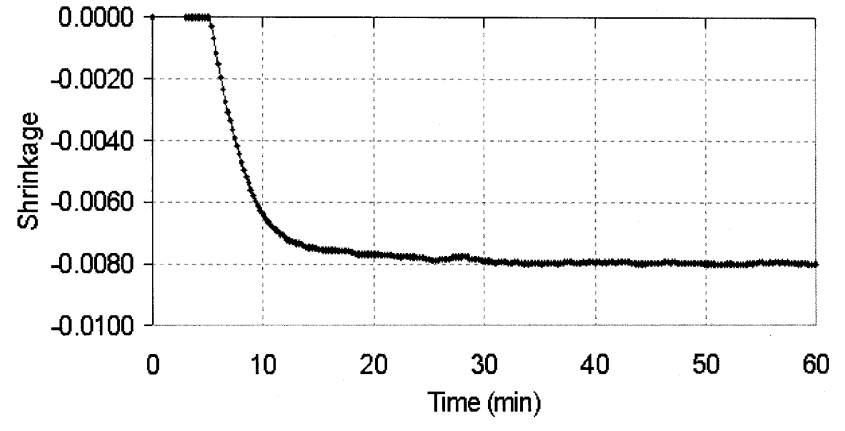

Fig. 1. Curing shrinkage evolution of the underfill epoxy.

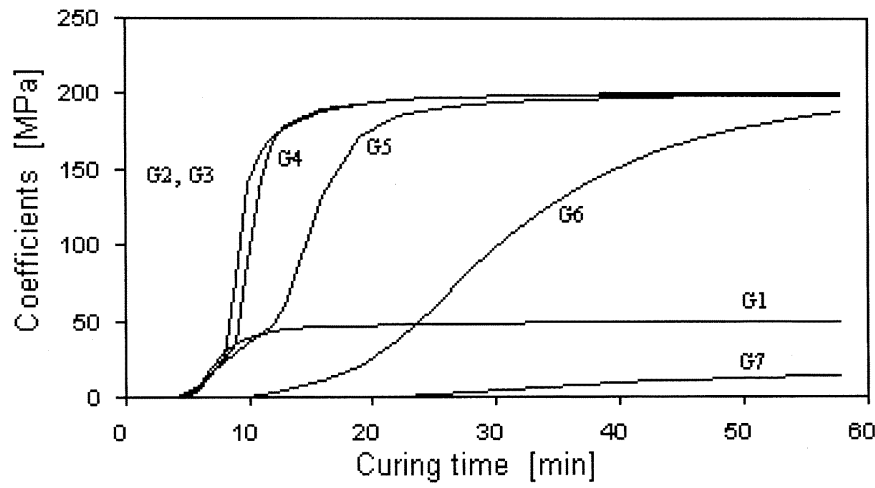

Fig. 2. Evolution of shear-stiffness functions $\left(G^{n}[\alpha(\xi)](n=1,2, \ldots, 7)\right.$, $\left.G^{8}[\alpha(\xi)] \sim 0\right)$ during cure.

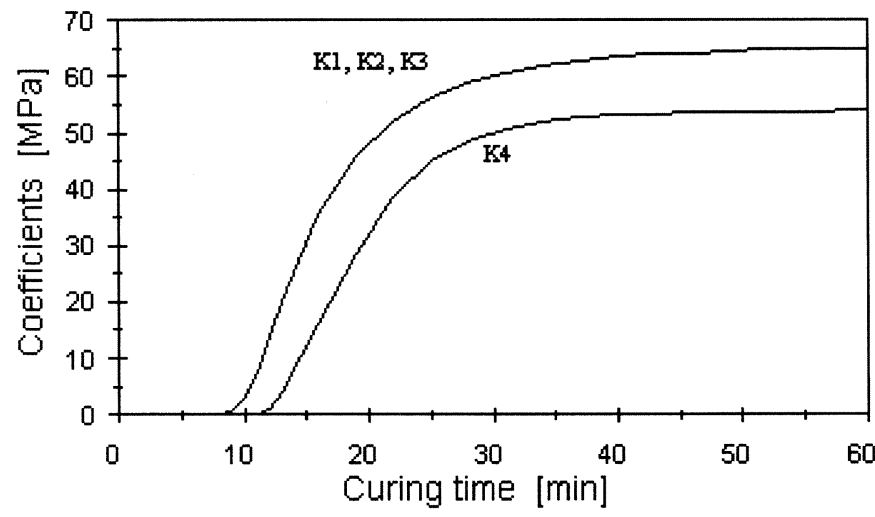

Fig. 3. Evolution of bulk stiffness functions $\left(K^{n}[\alpha(\xi)](n=1,2, \ldots, 4)\right.$, $K^{5} \sim K^{6} \sim K^{7} \sim 0, K^{8}=1000 \mathrm{MPa}$ ) during cure.

Here the curing shrinkage increment is represented by the initial strain increment $\Delta E_{j}^{*}\left(t_{m}\right)$.

Cure dependent viscoelastic parameters and chemical shrinkage strains are obtained through specially designed DMA measurements. The evolution of the curing shrinkage strain and the cure dependent stiffness coefficients for the used underfill epoxy are presented in Figs. 1-3, respectively. This epoxy, an acid anhydride system with a filler content of $63 \%$ amorphous $\mathrm{SiO}_{2}$, has an ultimate glass transition temperature of about $133{ }^{\circ} \mathrm{C}$ and is cured within $1 \mathrm{~h}$ at $130{ }^{\circ} \mathrm{C}$. More detailed description about the model and the experimental methodology can be found in [8]-[10].

Specially designed subroutines are applied for calculating the cure-dependent stiffness coefficients $K^{n}(\alpha)$ and $G^{n}(\alpha)$, and 
subsequently the stiffness functions $C_{i j}\left\{\alpha\left(t_{m}\right)\right\}$ and for transforming the shrinkage strain data to incremental initial strains. Verification of the model was presented in [9].

\section{EXPERIMENTAL}

\section{A. Description of Test Structures}

Accelerated temperature cycling tests were conducted to obtain failure data and to investigate failure mechanisms in FCOB assemblies. In order to investigate the effect of underfill, chip size and solder bump stand-off height on the fatigue life of a flip chip, a full factorial design-of-experiment (DOE) matrix was developed. Two levels were setup for each factor, i.e., underfill type $\mathrm{A}$ and $\mathrm{B}, 5 \times 5 \mathrm{~mm}^{2}$ and $10 \times 10 \mathrm{~mm}^{2}$ silicon test chips, $55 \mu \mathrm{m}$ and $82 \mu \mathrm{m}$ of stand-off high. Each test board had 32 test chips, on which the chips were distributed in such a way that all the geometric variables were used.

The $5 \times 5 \mathrm{~mm}^{2}$ test chip contains 48 solder bumps and the $10 \times 10 \mathrm{~mm}^{2}$ test chip contains 112 solder bumps. The solder bumps are distributed in two rows around the periphery of the die in a staggered configuration, as shown in Fig. 4. The pitch of the staggered configuration is $300 / 600 \mu \mathrm{m}$ for both sizes of chips. The solder bumped test chips were reflowed to the $1.6 \mathrm{~mm}$ thick FR-4 substrate. Afterwards, the assemblies were applied with underfill and cured at $130{ }^{\circ} \mathrm{C}$ for the amount of time specified by the manufacturer.

\section{B. Thermal Cycling}

After underfilling and curing process, the test assemblies were subjected to thermal cycling from $-55^{\circ} \mathrm{C}$ to $80{ }^{\circ} \mathrm{C}$, with a ramp rate of $10^{\circ} \mathrm{C} / \mathrm{min}$ and $5 \mathrm{~min}$ dwell time at each extreme. The upper temperature limit of the cycle was chosen below the glass transition temperature of the underfill and also not to cause overstress delamination in the interface of underfill and die-passivation.

During thermal cycling, the resistance of each daisy-chained flip chip assembly was measured and monitored real-time for failure. A failure was defined as the occurrence of fifteen transient electrical disturbances recorded by the event detector. The threshold resistance of the event detector was set to $300 \Omega$ for $t \geq 200$ nsec, since the maximum resistance of the flip chip assembly was below $30 \Omega$ before the thermal cycling.

After completing the thermal cycling test, a Weibull analysis was conducted to characterize the failure data. In addition, scanning electron microscopy (SEM) analyses on cross sections of related solder bumps were carried out to analyze the failure mechanisms.

\section{FE Modeling of the FCOB Package}

\section{A. Geometry and FEM Mesh}

A finite element thermo-mechanical modeling of a FCOB for fatigue predictions was carried out. The same FCOB configuration as used in the accelerated fatigue experiments is applied for the FEM modeling. For simplification, the passivation and metallization layer is ignored. Only the silicon die, the underfill, the solder bumps, the copper pads and the substrate are considered (see Fig. 4). In this paper, only one kind of used under-

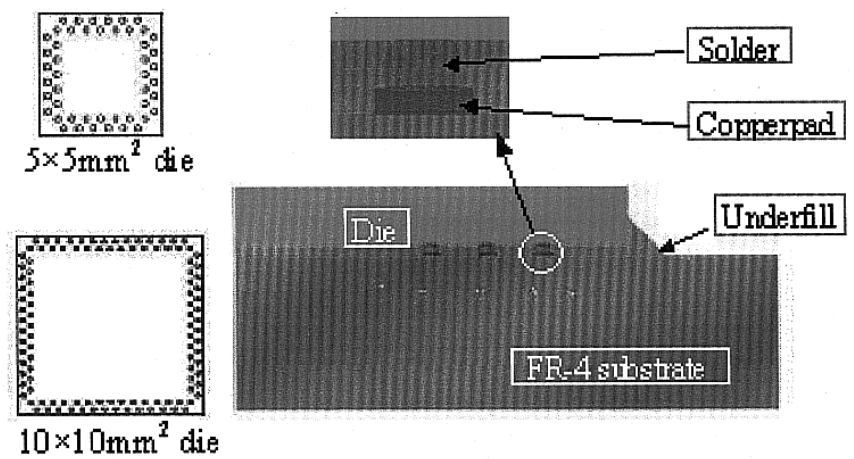

Fig. 4. Schematic illustration of the FCOB.

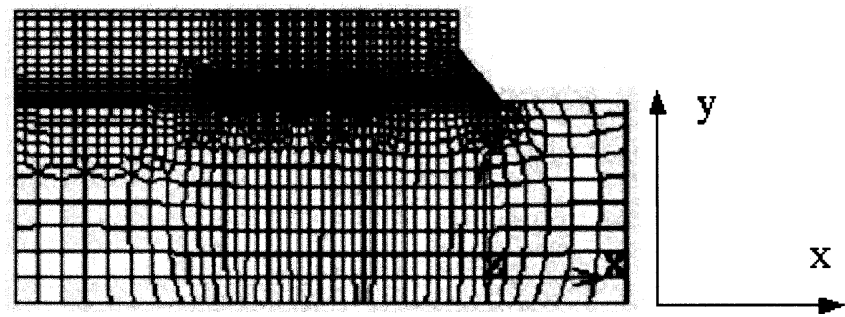

(a)

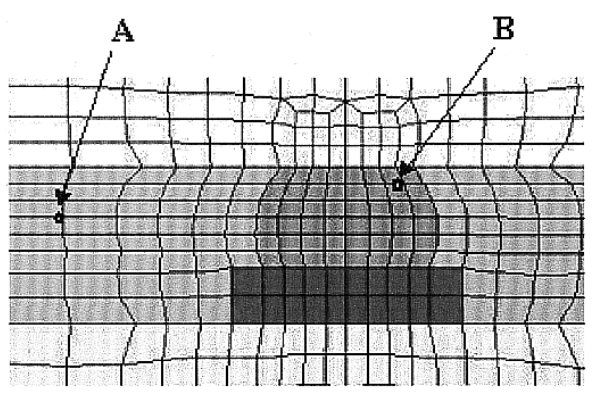

(b)

Fig. 5. FEM Mesh (a) mesh for package of $5 \times 5 \mathrm{~mm}^{2}$ die (b) close view of the bump, with indication of the specific locations (A, B).

fills, i.e., underfill "A" (HYSOL FP4526) is characterized for cure-dependent viscoelastic properties and subsequently used in the FEM-analyses. The underfill "A," a filled epoxy with a filler content of $63 \%$ (amorphous $\mathrm{SiO}_{2}$ ), has an ultimate glass transition temperature of about $133^{\circ} \mathrm{C}$. It is cured at $130^{\circ} \mathrm{C}$ for $60 \mathrm{~min}$.

2-D plane strain modeling is used. Because of the symmetry, only one half of the structure is simulated. Two FEM meshes are setup for both $5 \times 5 \mathrm{~mm}^{2}$ and $10 \times 10 \mathrm{~mm}^{2}$ chips. In order to facilitate a comparison between the results of both configurations, equivalent meshes are used around the solder bumps. Only one stand-off high, $82 \mu \mathrm{m}$ is considered. The FEM meshes are presented in Fig. 5. Following boundary conditions are used: the nodes along the symmetry axis are fixed in $\mathrm{x}$ direction $(u=0)$, and the node at the left bottom corner is fixed in $\mathrm{x}$ and $\mathrm{y}$ direction $(u=v=0)$.

\section{B. Thermal Loading}

The Flip Chip is subjected to a thermal loading equivalent to the fatigue test. At $130{ }^{\circ} \mathrm{C}$, the underfill is dispensed and cured for $60 \mathrm{~min}(\mathrm{a}-\mathrm{b})$, then the package is cooled down to 


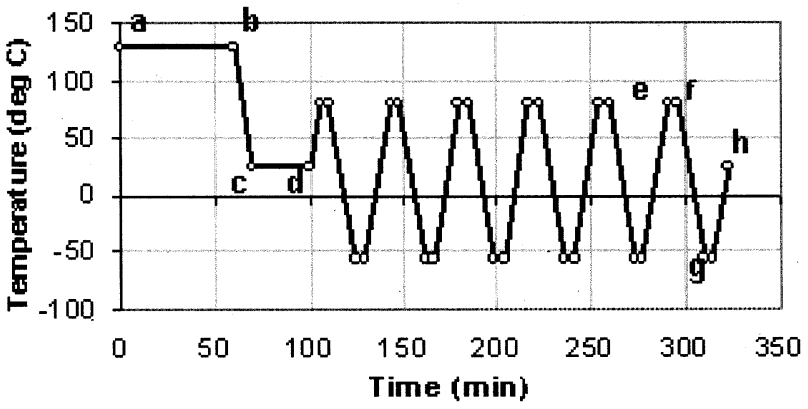

Fig. 6. Temperature profile.

room temperature at a ramp rate of $10{ }^{\circ} \mathrm{C} / \mathrm{min}$ and then being hold for $30 \mathrm{~min}(\mathrm{~b}-\mathrm{c}-\mathrm{d})$ and subsequently temperature cycles $(\mathrm{d}-\mathrm{e}-\mathrm{f}-\mathrm{g}-\mathrm{h})$ are applied. Fig. 6 shows the temperature profile with six thermal cycles used in the simulation. A stress-free situation is assumed at the curing starting point "a" for the so called "curing model."

In order to make a comparison between simulation results with and without the curing process being included, another two calculations are made for the $5 \times 5 \mathrm{~mm}^{2}$ and $10 \times 10 \mathrm{~mm}^{2}$ chips, now without consideration of the curing process. These have a loading history "b-c-d-e-f-h." For the "noncuring model," the stress-free state is assumed at the starting point " $\mathrm{b}$ " for all the materials in the package. It should be mentioned that more thermal cycles have been used for study on the stability of the strain ranges in the solder joints for both models as well.

\section{Material Properties}

The constitutive relation for curing resin as described before is implemented into the MARC FEM code and applied here for the simulation of the curing of the epoxy underfill. After curing, the material is considered to be temperature-dependent viscoelastic. Equations (1)-(4) are used, without the curing parameters. The time-temperature superposition principle is used to describe the temperature effect of the underfill resin during cooling down and thermal cycling. The temperature effect is accounted for by a reduced time, which is assumed to be same for all of the relaxation times in (3) and (4). It is defined by

$$
\zeta(t)=\int_{0}^{t} \frac{d \tau}{a_{T}(T(\tau))}
$$

where $a_{T}$ is the shift factor. It can be approximated by the wellknown Williams-Landel-Ferry (WLF) equation [12]

$$
\log a_{T}=\frac{-C_{1}\left(T-T_{r}\right)}{C_{2}+\left(T-T_{r}\right)}
$$

where $T_{r}$ is the reference temperature, $C_{1}$ and $C_{2}$ are constants. We choose the curing temperature $\left(130^{\circ} \mathrm{C}\right)$ as the reference temperature. Based on the DMA measurements on the cured samples with temperature scan from $-40^{\circ} \mathrm{C}$ to $150^{\circ} \mathrm{C}, C_{1}$ and $C_{2}$ are determined to be 36.8 and 576.5 , respectively.

The behavior of eutectic solder joints is modeled as temperature dependent elastic-plastic and separately rate-dependent creep. The temperature dependent Young's modulus is given by [13]

$$
E(T)=35366-151 T
$$

\begin{tabular}{|c|c|c|c|c|c|}
\hline \multicolumn{2}{|c|}{ Materials } & $\begin{array}{c}\mathrm{E} \\
(\mathrm{GPa})\end{array}$ & $\psi$ & $\begin{array}{c}\mathrm{G} \\
(\mathrm{GPa})\end{array}$ & $\begin{array}{c}\mathrm{CTE} \\
\left(\mathrm{ppm} /{ }^{\circ} \mathrm{C}\right)\end{array}$ \\
\hline \multicolumn{2}{|c|}{ Silican die } & 169 & 0.26 & -- & 2.3 \\
\hline \multicolumn{2}{|c|}{ Copper Pad } & 82.7 & 0.30 & -- & 16.7 \\
\hline \multirow[b]{2}{*}{ FR-4 } & In plane & 19.7 & 0.18 & 3.7 & 17.6 \\
\hline & $\begin{array}{l}\text { Out of } \\
\text { plane }\end{array}$ & 9 & 0.39 & 2.9 & 54.2 \\
\hline
\end{tabular}

TABLE I

MATERIAL PROPERTIES

where $\mathrm{T}$ is the temperature in ${ }^{\circ} \mathrm{C}$. The yield behavior is described by the following equation [14]:

$$
\sigma_{\mathrm{y}}(T)=49.2-0.097 T
$$

where $\mathrm{T}$ is the absolute temperature $(K)$.

The creep behavior is described as [13]

$$
\dot{\varepsilon}_{c r}=C_{1}\left(\frac{E}{T}\right) \cdot\left[\sinh \left(\frac{\alpha \sigma}{E}\right)\right]^{n} \cdot \exp \left(\frac{-Q}{K T}\right)
$$

where

$$
\begin{array}{ll}
\dot{\varepsilon}_{c r} & \text { equivalent creep strain rate; } \\
\sigma & \text { equivalent stress; } \\
E & \text { Young's modulus; } \\
Q & \text { activation energy; } \\
K & \text { universal gas constant; } \\
T & \text { absolute temperature; } \\
C_{1}, \alpha, n & \text { material constant. }
\end{array}
$$

The silicon die and copper pads are assumed to be isotropic, and the FR-4 substrate is considered to be orthotropic. Both are assumed to be temperature-independent and elastic during the loading history. The material properties used in the simulation are shown in Table I.

\section{Solder Fatigue Prediction Models}

Though there are quite a few solder fatigue prediction models available [1], so far it is still a confusing task to choose an appropriate model for prediction of fatigue life of a Flip Chip package, since most of the models and their parameters were obtained under specific test conditions and strain/stress states. In the considered FCOB package, the solder joints undergo both plastic and creep strains in the curing process as well as in the subsequent thermal cycles. Therefore, both the plastic and the creep strains should be taken into account for the fatigue prediction.

A Coffin-Manson based relationship, proposed by Solomon [15], has been successfully applied to underfilled flip chip, for instance, by Pang et al. [2], [14]. The model relates fatigue life cycles to the plastic shear strain range, as shown in

$$
N_{p}=C\left(\Delta \gamma_{p}\right)^{\eta}
$$

where $\Delta \gamma_{p}$ represents the plastic shear strain range, $N_{p}$ represents the number of cycles to failure, and $C$ and $\eta$ are constants. Based on measurements at $-50^{\circ} \mathrm{C}, 35^{\circ} \mathrm{C}$, and $125^{\circ} \mathrm{C}$, Solomon [15] reported the value of $C$ and $\eta$ for $\mathrm{Sn}-\mathrm{Pb}$ eutectic solder. The averaged values of $C$ and $\eta$ are 1.2928 and -1.96 , respectively.

It is commonly accepted that creep may be due to matrix creep and grain boundary sliding. Knecht and Fox [16] have proposed 
a fatigue model relating the fatigue life with the solder microstructure and the creep shear strain range due to matrix creep

$$
N_{c r}=\frac{C}{\Delta \gamma_{m c}}
$$

in which $N_{c r}$ is the number of cycles to failure. $\Delta \gamma_{m c}$ is the shear strain range due to matrix creep. $\mathrm{C}$ is dependent on failure criteria and the solder microstructure, which is $890 \%$. Shine and Fox [17] reported that fatigue life due to creep strains shows much correlation to matrix creep but relatively little correlation to grain boundary creep. Thus, the effect on the creep strains due to the grain boundary sliding of the solder bumps is neglected in this paper.

By applying Miner's linear superposition principle, a strainbased fatigue model, which combines the plastic and creep effect, is formulated by [2]

$$
\frac{1}{N_{f}}=\frac{1}{N_{p}}+\frac{1}{N_{c r}}
$$

where $N_{f}$ represents the number of cycles to failure. $N_{p}$ and $N_{c r}$ refer to the number of cycles to failure due to plastic fatigue and creep fatigue, respectively.

Because these fatigue models were based on a pure shear state, the equivalent plastic strain and equivalent creep strain should be substituted for the shear strains

$$
\gamma_{e q}=\sqrt{3} \varepsilon_{e q} .
$$

Equations (12)-(14) are applied in this paper as indicator for the fatigue failure prediction. It should be noted that because only equivalent strain ranges are used in the above models, the effect of the hydrostatic stress in the solder has been not included.

\section{RESULTS AND DISCUSSIONS}

\section{A. Curing-Induced Stress and Strain}

Fig. 7 shows the evolution of the horizontal stress component $\sigma_{\mathrm{xx}}$ at point $\mathrm{A}$ in the underfill and the equivalent von Mises stress at point $\mathrm{B}$ in the outmost solder bump during the curing process. The locations of point $\mathrm{A}$ and $\mathrm{B}$ as well as the coordinate system are illustrated in Fig. 5. Fig. 8 presents the evolution of the horizontal strain component and the cure-induced shrinkage strain in the underfill, and the equivalent plastic strain and equivalent creep strain in the solder bump during the curing process as well.

During the curing process, the constrained shrinkage and the simultaneously increasing stiffness of the underfill resin cause the stresses in the package. The shrinkage of underfill is constrained by the adjacent materials in the package. Adversely, the solder bumps are deformed and stressed by the shrinkage. Meanwhile, the stress relaxation behavior in the underfill and the plastic deformation and creep in the solder bumps also influence the evolution of stresses during cure and thus influence the final residual stress state.

After starting the curing for about $4.5 \mathrm{~min}$, an intense crosslinking reaction occurs in the underfill after passing the gelation point. The stresses in the package then increase quickly. At about 15 min, most of the crosslinking is finished. Plastic deformation takes place in some local parts with high

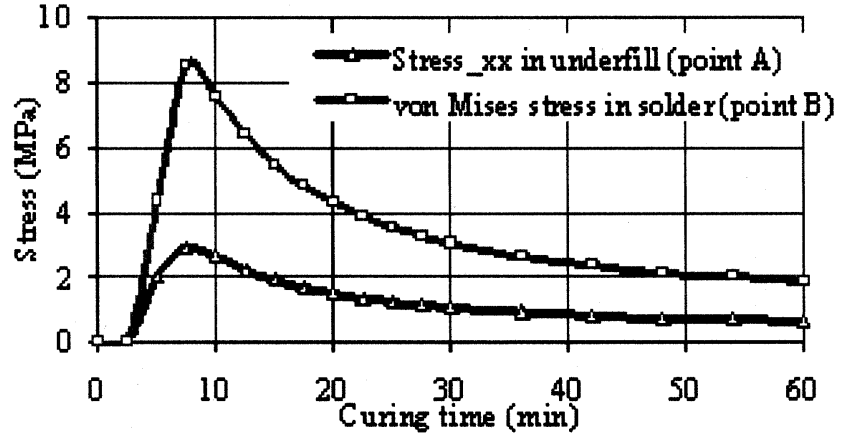

Fig. 7. Stress $\sigma_{\mathrm{xx}}$ evolution in underfill (point A) and equivalent von Mises stress in the solder bump (point B) during cure.

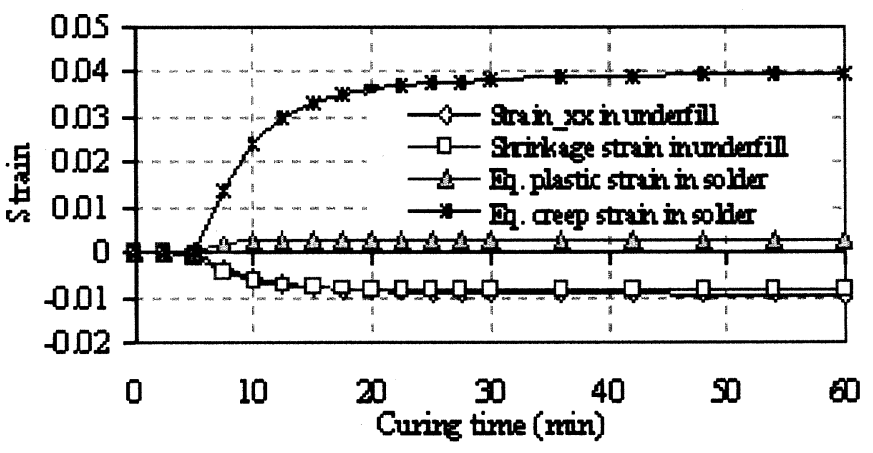

Fig. 8. Strain history in underfill (point A) and in the solder bump (point B) during cure.

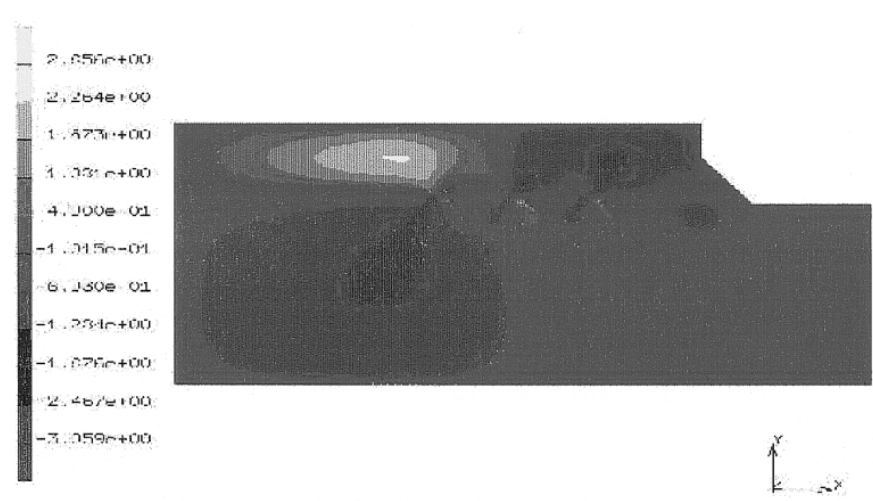

Fig. 9. Residual shear stress distribution in the package at the end of curing $(t=60 \mathrm{~min})$.

stresses in the solder bumps. Subsequently, the stress relaxation behavior in the underfill and creep in the solder bumps prevail over the chemical shrinkage of the underfill. The stresses in the underfill and the solder bumps decline dramatically. Relatively low stress levels are found in the underfill and the solder bumps at the end of curing. However, a high level of creep strain is found in the bumps. It can be seen that the creep strain is nearly an order larger than the plastic strain for a large part of the bump.

Fig. 9 represents the contour plot of the shear stress in the whole package at the end of curing. Relatively high stress levels are found in the underfill, the solder bumps and the silicon die.

Fig. 10(a)-(c) shows the contour plots of the equivalent creep strain in the outermost solder bump at curing time $t=125 \mathrm{~min}$, the end of curing and subsequent cooling down to room temprature. Fig. 10(d) shows the distribution of equivalent creep strain when directly cooling down from $130{ }^{\circ} \mathrm{C}$ to room tem- 


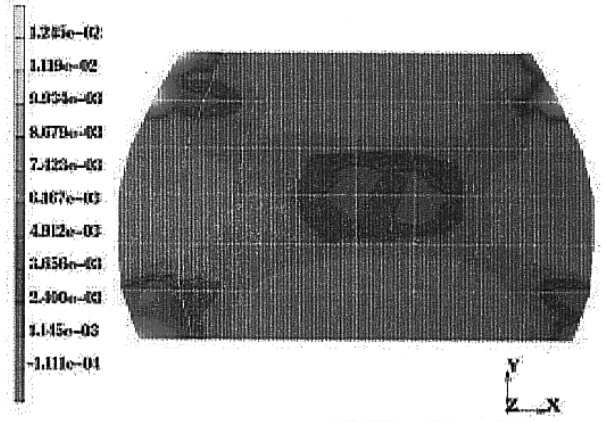

(a)

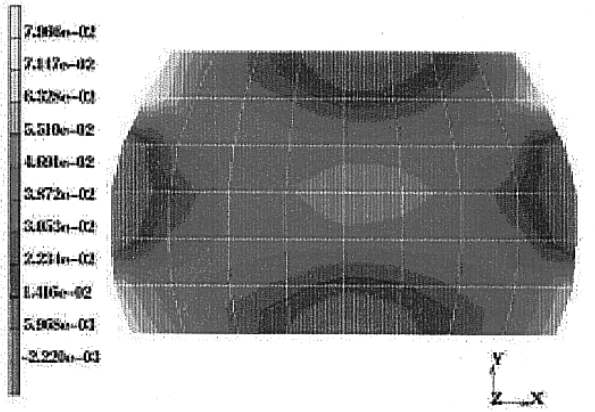

(c)

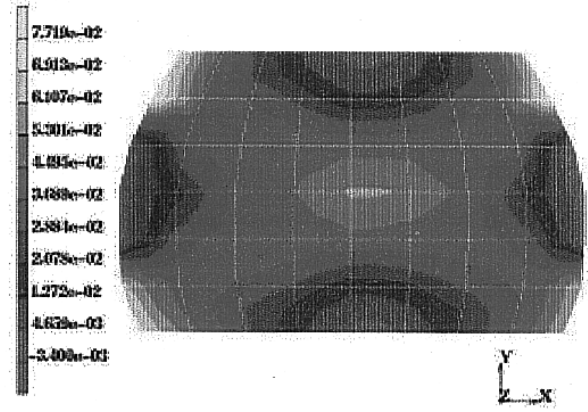

(b)

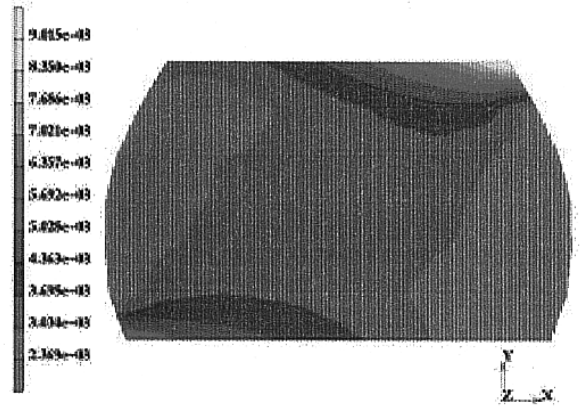

(d)

Fig. 10. Equivalent creep strain evolution in solder bump: (a) at curing time $t=12.5 \mathrm{~min}$, (b) at the end of curing $t=60 \mathrm{~min}$, (c) subsequent cooling dowbn to room temprature, and (c) Directly cooling down to room temperature from $130^{\circ} \mathrm{C}$ without consideration of curing.
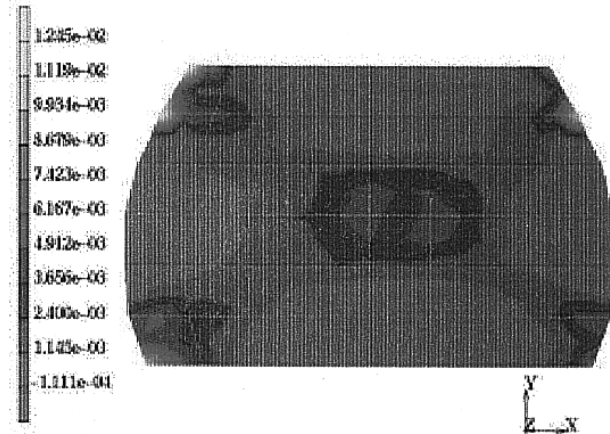

(a)

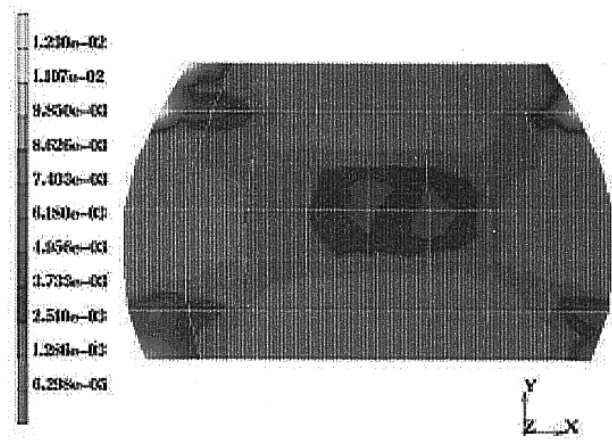

(c)

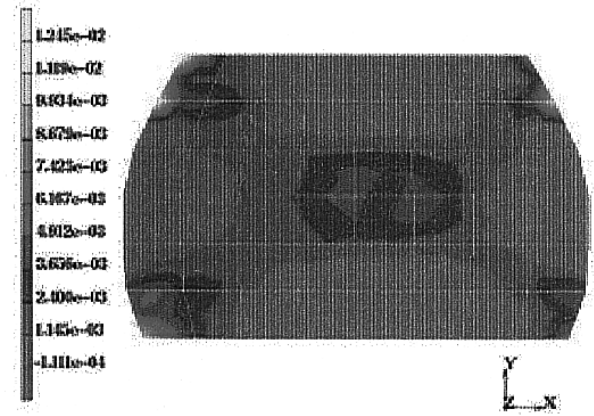

(b)

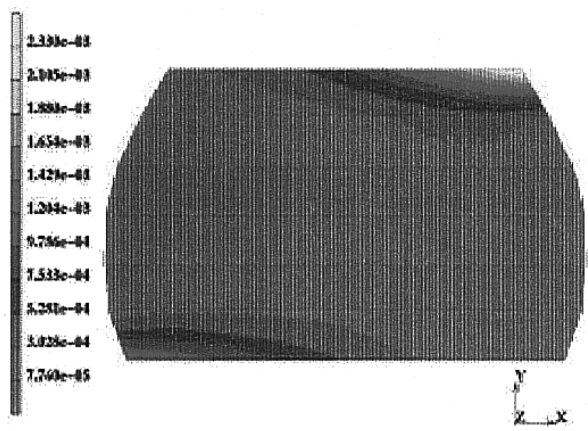

(d)

Fig. 11. Equivalent plastic strain evolution in solder bump: (a) at curing time $t=12.5 \mathrm{~min}$, (b) at the end of curing $t=60 \mathrm{~min}$, (c) subsequent cooling dowbn to room temprature, and (d) directly cooling down to room temperature from $130{ }^{\circ} \mathrm{C}$ without consideration of curing.

perature without consideration of curing. It is found that very high creep strain level has been in the solder during the curing process. The distribution and the level of the creep strain caused by the curing process is different from that caused by thermal shrinkage. The thermal shrinkage strain is more concentrated in the corners of the bump, however, in the case of considering the curing process, the distribution of the strain in the bump is much more uniform. Similarily, Fig. 11 shows the contour plots of the equivalent plastic strain at different process phases. Therefore, in FEM modeling, the curing process has a significant effect on 


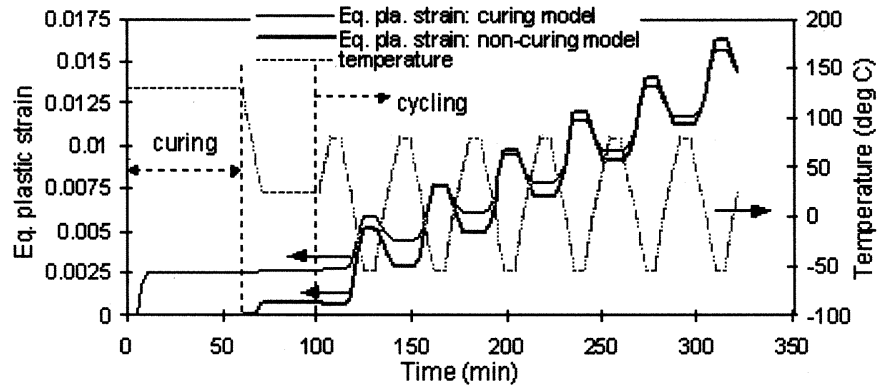

Fig. 12. History of the equivalent plastic strains and the temperature profile with and without the curing process included.

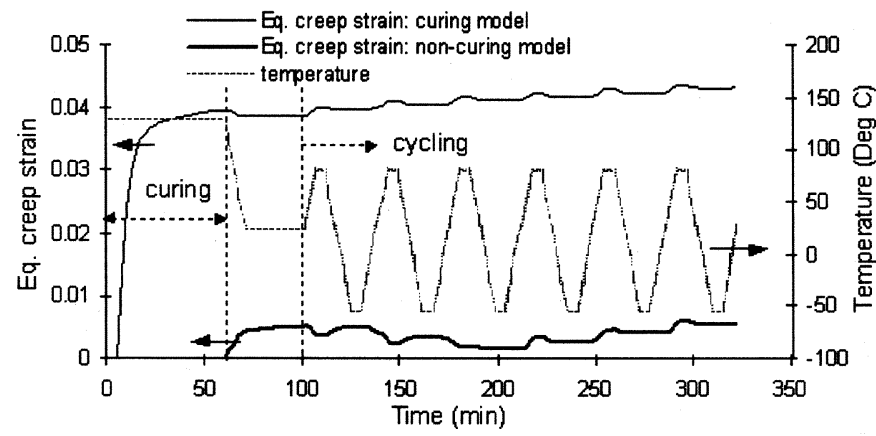

Fig. 13. History of the equivalent creep strains and the temperature profile with and without the curing process included.

both the plastic and the creep strains of the solder bumps, thus certainly influencing the fatigue life prediction results.

\section{B. Experimental Fatigue and Model Prediction Results}

Only the test results associated with underfill " $\mathrm{A}$ " are presented in this paper. The fatigue test results reveal that the stand-off height of solder bumps has only a slight effect on the durability of the FCOB compared to the chip size. So its influence is neglected in this paper. The mean times to failure of the $5 \times 5 \mathrm{~mm}^{2}$ chip and the $10 \times 10 \mathrm{~mm}^{2}$ chip are $6440 \mathrm{~h}$ and 5750, respectively. The matching mean numbers of cycles to failure are 10443 and 9324 cycles, respectively. The ratio of mean life of the $5 \times 5 \mathrm{~mm}^{2}$ chip versus the $10 \times 10 \mathrm{~mm}^{2}$ chip is about 1.12 .

The outermost bump in the package is selected for further failure prediction considerations. These values are averaged over the elements in the upper right corner, representing about $8 \%$ of the bump volume. Fig. 12 shows the time history of the equivalent plastic strains for six thermal cycles with and without consideration of the curing process in the solder bump of the $5 \times 5 \mathrm{~mm}^{2}$ chip package, together with the temperature profile for clarification. During the curing process, the magnitude of the equivalent plastic strain generated is about $0.25 \%$. Similarly, Fig. 13 presents the time history of the equivalent creep strains with and without the curing process included. It is seen that solder bumps undergo a very high level of creep stain during the curing process, as high as $4 \%$, more than one order higher than the plastic strain. This is due to the fact that the solder bumps are exposed to a high temperature when the curing-induced stresses of the underfill are high. During the phase of cooling down from $130^{\circ} \mathrm{C}$ and dwell time, a different effect on the creep strains is seen if the curing process is

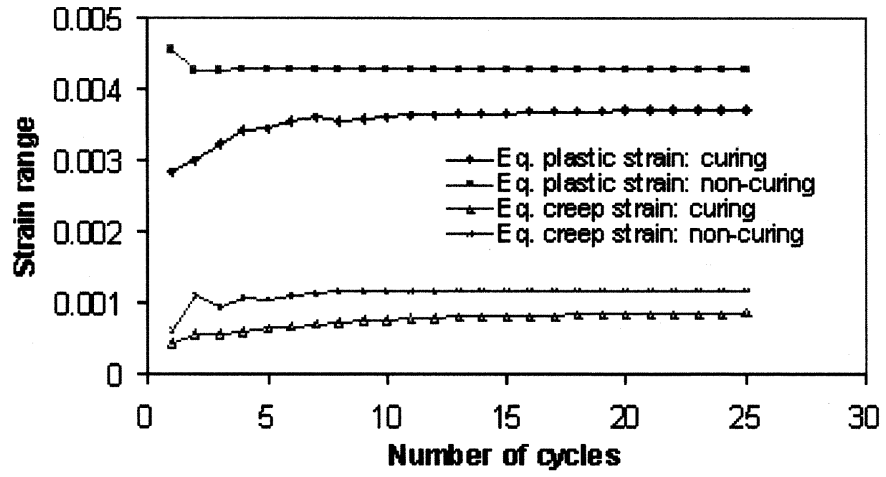

Fig. 14. Variation of the strain ranges versus number of thermal cycles.

TABLE II

Equivalent Strain Ranges For THE $5 \times 5 \mathrm{~mm}^{2}$ ChIP-Size FCOB AT THE 25TH CYCLE

\begin{tabular}{c|c|c}
\hline Strain range & Curing & Non-curing \\
\hline$\Delta \varepsilon_{\text {eq_F }}$ & $0.3708 \%$ & $0.4277 \%$ \\
\hline$\Delta \varepsilon_{\text {eq_a }} \sigma$ & $0.0825 \%$ & $0.1152 \%$ \\
\hline
\end{tabular}

considered. The creep strain level is decreased. During thermal cycles, the creep strain increases as time increases. In the case of the curing process not included, during first three cycles, the creep strain decreases in whole as the time increases. The reason maybe is that when the solder is cooled down to room temperature, a very high stress is concentrated at the corner part and in the first few cycles, the creep strain caused by the thermal cycling counteracts a part of the creep strain caused by the cooling down. As the thermal cycling proceeds, the deformation is more uniform and the deformation of the solder bumps becomes more stable. After three cycles, the creep strain increases as the time increases, with a higher strain range than that with consideration of the curing process.

In a previous paper [18], the strain ranges of the third cycle were taken for calculation of the fatigue life. It may not be appropriate because it may need longer time for the strain ranges in the solder to become stable if the curing process is included in the simulation. In order to study the stability of the strain ranges, more thermal cycles are adapted for current simulations. Fig. 14 illustrates the variation of both equivalent plastic and creep strain ranges from the first cycle to 25 th thermal cycle. It is seen that with the noncuring model, the strain ranges are nearly stable after six cycles, but with the curing model, more cycles are needed to get stable strain ranges for fatigue prediction.

Table II lists the equivalent plastic strain range and equivalent creep strain range during the 25th thermal cycle for the $5 \times 5 \mathrm{~mm}^{2}$ chip-size package. By using (12)-(14), the number of cycles to failure relating to plastic strain, creep strain and the combination of both can be calculated. Table III presents the number of cycles to failure with and without consideration of the curing process, respectively.

It is found that Solomon's plastic strain fatigue model predicts a much higher fatigue life than Knecht and Fox's creep strain model. That means that creep fatigue plays a predominant role in the whole fatigue life of the solder bumps. 
TABLE III

Lists OF NUMBER OF CYCLES TO FAILURE, EXPERIMENTAL AND MODEL PREDICTION RESUlTS FOR THE $5 \times 5 \mathrm{~mm}^{2}$ CHIP-SizE FCOB AT THE 25TH CYCLE

\begin{tabular}{c|c|c}
\hline Fatigue model & Curing & Non-curing \\
\hline $\begin{array}{c}\text { Plastic strain } \\
\text { model eq. (12) }\end{array}$ & 25610 & 19357 \\
\hline $\begin{array}{c}\text { Creep strain } \\
\text { model eq. (13) }\end{array}$ & 6228 & 4462 \\
\hline $\begin{array}{c}\text { Plastic \& creep } \\
\text { model eq. (14) }\end{array}$ & 5010 & 3626 \\
\hline Experimental & \multicolumn{2}{|c}{10443} \\
\hline
\end{tabular}

The curing process has influence on the fatigue prediction results of all the fatigue models. From Tables II and III it is indicated that if the curing process is considered in the FEM modeling, the predicted fatigue life is higher than that without consideration of curing. However, it still underestimates the fatigue life compared with the experimental results. One of the main reasons for the under-estimation is that the used fatigue prediction models (12)-(14) only account for the deviatoric part of the loaded stress and strain.

The effects of the curing process induced stress/strain on the fatigue life time of the solder joints may be explained in two aspects. One is the effect resulted from the deformation and residual stresses in the whole package, i.e., the global effect. The other is the effect of the cure-induced residual stress/strain in the bump itself, the so called local effect.

Fig. 15(a) and (b) show the deformation of the whole package at the end of curing and at the high extreme of the sixth cycle $\left(80^{\circ} \mathrm{C}\right)$ (corresponding to "e" in Fig. 6), respectively. The deformation caused by the curing shrinkage is obviously different with the deformation caused by thermal stress. Cure shrinkage causes the package, especially the die, to bend upwards, resulting compressive stress at the centre part of the die backside. Simulation results indicate that the die deflection during thermal cycling is lower when the curing process is taken into account, which is favorable to the solder bump fatigue life time. The residual stress/strain will counteract a part of deformation and stress during the subsequent thermal cycling. For instance, because the plastic behavior of the solder is modeled as elastic and perfectly plastic, when the von Mises stress in some part or whole of the solder reachs the yield stress at certain temperature during cycling, the von Mises stress will not change. But the plastic strain in this part may contiune to change due to the deformation of the adjacent materials. That probaly is the reason why the plastic range calcultaed from the simulations is higher than that with consideration of the curing process. Although part of the global effect will relax during curing, cooling down and thermal cycling because of the viscoelastic property of the underfill, it will reach an equilibrium state determined by the equilibrium moduli. Therefore, by including the curing process in the simulation, the effect of the residual stress and deformation in the whole package on the solder stress/srain and fatigue life prediction, i.e., the global effect, can be accessed.

As for the curing-induced stress field in the solder bumps (the local effect) caused by the cure shrinkage of underfill, it can be

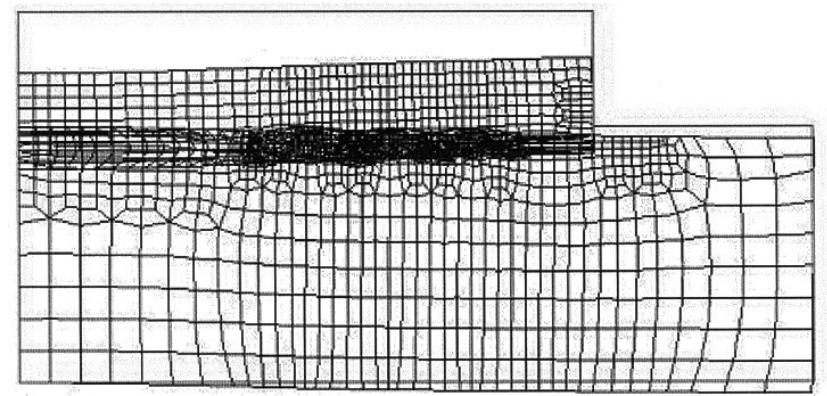

(a)

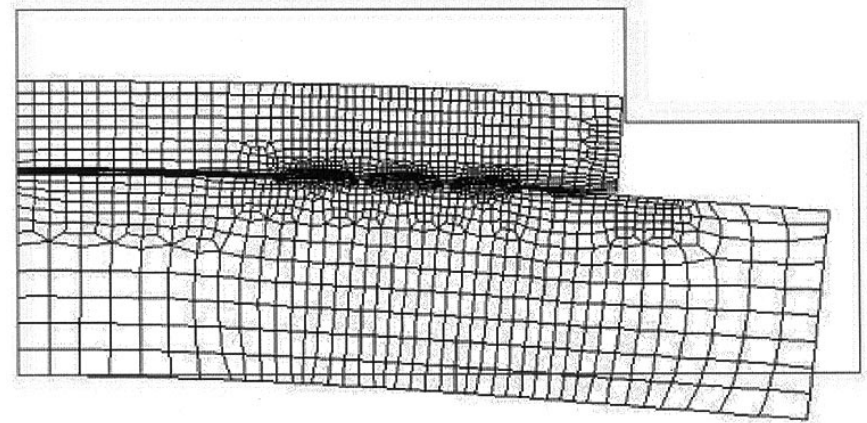

(b)

Fig. 15. Deformation of the package: (a) at the end of curing $(t=60 \mathrm{~min})$ and (b) at the high extreme $\left(80^{\circ} \mathrm{C}\right)$ of the sixth cycle $(t=291 \mathrm{~min})$.

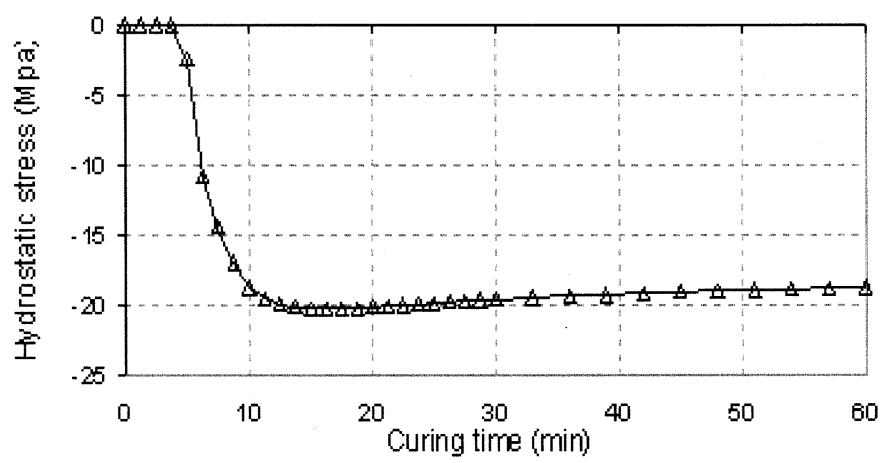

Fig. 16. Evolution of the hydrostatic stress in the solder during the curing process.

decomposed into a deviatoric part and a hydrostatic part. The effect of the hydrostatic part will be discussed in Section VI-C. In reality, the deviatoric part will be relaxed over time due to creep. In our simulation, as shown in Fig. 7, it can be seen that the level of von Mises stress in the solder bump decreases from the highest point as the curing proceeds. Till the end of curing, more than $80 \%$ of the von Mises stress has been relaxed due to the creep behavior of the solder bump and relaxation of underfill as well. During cooling down and subsequent cycles, it will further be relaxed and more cycles are needed in simulations to get stabilized strain ranges.

\section{Effect of Curing-Induced Hydrostatic Stress}

Compressive hydrostatic stress is induced in the solder bumps during the curing process because of the crosslinking shrinkage of the underfill. Fig. 16 shows the evolution of the averaged hydrostatic stress in the considered part of the solder bump. It 
decreases slightly from the peak point due to the relaxation of the underfill. At the end of curing, it has a value of $18.7 \mathrm{Mpa}$.

Although the deviatoric part of the cure-induced stress in the solder will eventually be relaxed, the compressive hydrostatic stress does not relax with time due to solder creep. It is commonly accepted that such a compressive hydrostatic stress is favorable for the solder fatigue life because it possibly hinders void nucleation or crack initiation or tends to close microcracks during thermal cycling. Or it will decrease the maximum tensile stress during the low temperature parts of thermal, which may result in a slow propagation of a crack and thus it is beneficial to the fatigue life. Furthermore, it is reported by Dutta et al. [19] that the imposition of compressive hydrostatic stress on the solder joint can effectively reduce coarsening, which significantly influence the strain response. Unfortunately, the fatigue prediction models (12)-(14) used early, which only account for the deviatoric part, i.e., the equivalent strains, are unable to take this effect into account. So far, no well-developed models which have considered the effect of the hydrostatic stress are available for solder.

Darbha et al. [5] modified the energy partitioning damage model by using a correction factor in the creep damage formulation to take into account the effect of the hydrostatic stress. Assuming that plasticity is not affected by the hydrostatic stress, when a constant hydrostatic stress is applied on the solder, the number of cycles to failure due to creep damage can be computed by

$$
\frac{1}{N_{f c}}=\left(\frac{W_{c}}{W_{c o}\left[1-\frac{\sigma_{H}}{\sigma_{f}}\right]^{n}}\right)^{-1 / d^{\prime}}
$$

where

$N_{f c}$ number of cycles to failure due to creep;

$W_{c} \quad$ creep work density per cycle;

$W_{c o}$ coefficient;

$\sigma_{H} \quad$ hudrostatic stress;

$\sigma_{f} \quad$ hudrostatic stress exponent;

$d^{\prime} \quad$ constant.

In [5] $W_{c o}, n$ and $d^{\prime}$ in (16) were obtained from the experimental results of a particular flip chip configuration. For a qualitative examination of the influence of the cure-induced hydrostatic stress, we use the values for $W_{c o}, n$ and $d^{\prime}$ provided in [5]. The hydrostatic stress at the end of curing is considered as an external pressure and to be constant during thermal cycling. By using (16), a comparison between with and without the hydrostatic stress can be made. It is indicated that the predicted number of cycles to failure due to creep is more than twice of that without the cure-induced hydrostatic stress included. Therefore, the effect of the cure-induced hydrostatic stress is significant for the fatigue prediction results of the FCOB package. However, it should be mentioned that it is a rough estimation and further investigation in modeling and experimental aspects is needed to appropriately incorporate the effect of hydrostatic stress into the fatigue prediction models.

Fig. 17 shows a SEM picture of a cross-section of one of the failed bumps. Cracks can be seen, starting at the top corners.

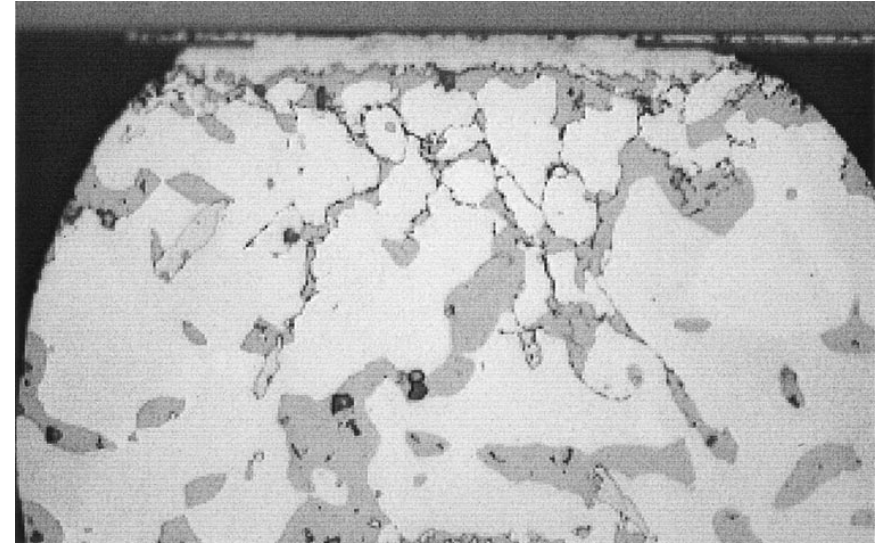

Fig. 17. SEM picture of cross section of a failed bump.

\section{CONCLUSION}

Accelerated fatigue experiments with thermal cycles from $-55^{\circ} \mathrm{C}$ to $80^{\circ} \mathrm{C}$ are carried out for a specially designed Flip Chip configuration. Based on a cure-dependent viscoelastic constitutive relation for the underfill epoxy, thermo-mechanical behavior of the tested FCOB during the curing process and the subsequent temperature cycling is modeled. The fatigue life predictions are based on simulations with and without considering the curing process.

The results show that the creep strain plays a predominant role on the fatigue life of the FCOB. In FEM modeling, the curing process has a significant effect on both the plastic and the creep strains of the solder bumps, thus influencing the fatigue life prediction results. It is indicated that if the curing process is considered in the FEM modeling, more thermal cycles need to be carried out to get stable strain ranges for fatigue life prediction. Fatigue prediction results, which are based on a combination of a Coffin-Manson based fatigue relation and a creep fatigue model reveals that the predicted fatigue life match better with the experimental results than that without consideration of curing for both chip-size packages, but it still underestimates the fatigue life. The main reason for this underestimation is that the used fatigue models are shear strain based and the effect of the hydrostatic stress has not been included.

Compressive hydrostatic stress is induced in the solder bump during the curing process. An estimation, based on a modified energy partitioning damage model with a correction factor in the creep damage formulation to take into account the effect of the hydrostatic stress, shows that such a hydrostatic stress is significant for the solder fatigue life. Therefore, the cure-induced residual stress/strain state should not be neglected in order to get reliable prediction results. Further investigation is needed to appropriately incorporate the effect of hydrostatic stress into the fatigue prediction models.

\section{REFERENCES}

[1] W. W. Lee, L. T. Nguyen, and G. S. Selvaduray, "Solder joint fatigue models: Review and applicability to chip scale packages," Microelectron. Rel., vol. 40, no. 2, pp. 231-244, 2000.

[2] J. Pang, T. I. Tan, and S. K. Sitaraman, "Thermo-mechanical analysis of solder joint fatigue and creep in a flip chip on borad package subjected to temperature cycling loading," in Proc. 48th IEEE Electron. Comp. Technol. Conf.(ECTC'48), 1998, pp. 878-883. 
[3] J. H. Okura, S. Shetty, B. Ramakrishnan, A. Dasgupta, J. F. J. M. Caers, and T. Reinikainen, "Guidelines to select underfills for flip chip on board assemblies and compliant interposers for chip scale package assemblies," Microelectron. Rel., vol. 40, no. 7, pp. 1173-1180, 2000.

[4] G. Q. Zhang and C. de Hoon, "Modeling of solder creep in electronic packages," Internal Rep., CFT/Philips, 1997.

[5] K. Darbha, J. H. Okura, S. Shetty, A. Dasgupta, T. Reinikainen, J. Zhu, and J. F. J. M. Caers, "Thermomechanical durability analysis of flip chip solder interconnects: Part 2-With underfill," ASME J. Electron. Packag., vol. 121, no. 4, pp. 237-241, 1999.

[6] M. S. Kiasat, A. H. J. Nijhof, H. Blokland, and R. Marissen, "Shrinkage and stress build up in unsaturated polyester resin during curing," Polymers Ceramics, vol. 2, pp. 95-102, 1997.

[7] M. S. Kiasat, "Curing shrinkage and residual stress in viscoelastic thermosetting resins and composites," Ph.D. dissertation, Delft Univ. Technol., Delft, The Netherlands, 2000.

[8] L. J. Ernst, C. van't Hof, D. G. Yang, M. S. Kiasat, G. Q. Zhang, H. J. L. Bressers, J. F. J. Caers, A. W. J. den Boer, and J. Janssen, "Mechanical characterization and simulation of curing packaging polymers," in Proc. 3th Int. Conf. Micro Mater., Berlin, Germany, 2000, pp. 162-167.

[9] C. van't Hof, D. G. Yang, M. S. Kiasat, L. J. Ernst, G. Q. Zhang, H. J. L. Bressers, J. F. J. Caers, A. W. J. d. Boer, and J. Janssen, "ThermoMechanical characterization of packaging polymers during the curing process," in Proc. Workshop Polymeric Mater. Microelectron. Photon. Applicat.: Mech., Phys., Rel., Process., EPP, vol. 27, Paris, France, Dec. 1999, pp. 153-158.

[10] L. J. Ernst, C. van't Hof, D. G. Yang, M. S. Kiasat, G. Q. Zhang, H. J. L. Bressers, J. F. J. Caers, A. W. J. den Boer, and J. Janssen, "Mechanical modeling and characterization of the curing process of underfill materials," J. Electron. Packag., Trans. ASME, vol. 124, no. 2, pp. 97-105, 2002.

[11] D. G. Yang, L. J. Ernst, C. van't Hof, M. S. Kiasat, J. Bisschop, J Janssen, F. Kuper, Z. N. Liang, R. Schravendeel, and G. Q. Zhang, "Vertical die crack stresses of flip chip induced in major package assembly processes," Microelectron. Rel., vol. 40, pp. 1533-1538, 2000.

[12] J. Aklonis and W. J. MacKnight, Introduction to Polymer Viscoelasticity, 1983.

[13] R. Darveaux and K. Banerji, "Constitutive relations for tin-based solder joints," IEEE Trans. Comp., Hybrids, Manufact. Technol., vol. 15, pp. 1013-1024, June 1998

[14] J. Pang, T. I. Tan, Y. R. Chong, G. Y. Lim, and C. L. Wong, "Analysis of underfill encapsulation curing deformation on flip chip on board (FCOB) package reliability," J. Electron. Manufact., vol. 8, no. 3, pp. 181-191, 1998

[15] H. D. Solomon, "Fatigue of 60/40 solder," IEEE Trans.Comp., Hybrids, Manufact. Technol., vol. 9, pp. 423-432, 1986.

[16] S. Knecht and L. R. Fox, "Constitutive relation and creep-fatigue life model for eutectic tin lead solder," IEEE Trans. Comp., Hybrids, Manufact. Technol., vol. 13, pp. 424-433, June 1990.

[17] M. C. Shine and L. R. Fox, "Fatigue of solder joints in surface moun devices," in Proc. Low Cycle Fatigue, ASTM STP 942, H. D. Solomon, Ed., Philadelphia, PA, 1988, pp. 588-610.

[18] D. G. Yang, G. Q. Zhang, L. J. Ernst, J. F. J. Caers, H. J. L. Bressers, and J. Janssen, "Combined experimental and numerical investigation on flip chip solder fatigue with cure-dependent underfill properties," in Proc. 51th IEEE Electron. Comp. Technol. Conf. (ECTC'51), 2001, pp. 919-924.

[19] I. Dutta, A. Gopinath, and C. Marshall, "Underfill constraint effects during thermomechanical cycling of flip-chip solder joints," J. Electron. Mater, vol. 31, no. 4, pp. : 253-264, 2002.

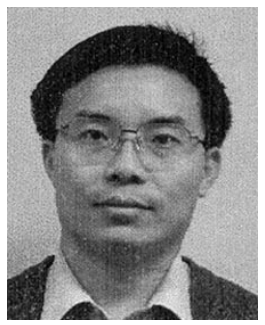

D. G. Yang received the M.Sc. degree in engineering mechanics from Zhejiang University, Hangzhou, China, in 1989 and is currently pursuing the Ph.D. degree at the Delft University of Technology, Delft, The Netherlands.

He worked in Guilin University of Electronic Technology, China, since 1989 as a Lecturer and Associate Professor. His research interests are in the areas of finite element modeling, experimental mechanics, reliability of microelectronic packaging and assembly, (micro-) damage and failure mechanism of electronic packaging, and interconnection materials.

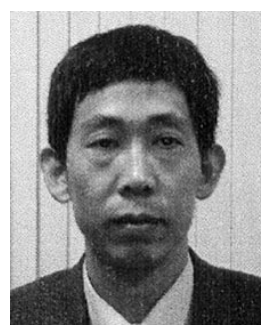

G. Q. Zhang is a Professor at the Faculty of Mechanical Engineering, Eindhoven University of Technology, Eindhoven, The Netherlands, and Principal Scientist and Competence Coordinator with the Center for Industrial Technology (CFT)/Philips, Eindhoven, the Netherlands. $\mathrm{He}$ is author and co-author for more than 80 scientific publications, including journal and conference papers, books, and invited keynote lectures. Besides his responsibilities in the Eindhoven University of Technology, he is also an Invited Ph.D. and Postdoc Supervisor with other universities, such as Delft University of Technology. His scientific interest include computational mechanics, experimental mechanics, and optimization methods, especially their applications in microelectronics and nanotechnology.

Dr. Zhang is General Chair of of the International Conference EuroSimE, and a member of committees of several international conferences and scientific societies.

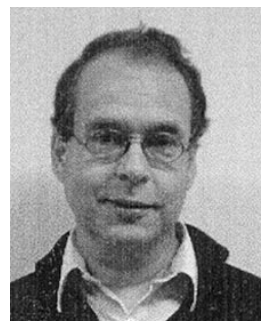

Leo J. Ernst received the Ph.D. degree in technical sciences from the Delft University of Technology, Delft, The Netherlands, in 1981.

$\mathrm{He}$ is a Professor and the head of the Engineering Mechanics Research Group, Mechanics and Control Department, Delft University of Technology. He has worked in various fields of application. In the last five years, he focused on mechanics of microelectronics. He has authored and co-authored over 150 professional and scholarly publications in the very specialized field of engineering mechanics.

Dr. Ernst is the acting Technical Chair for EuroSIME Conferences on "Thermal and Mechanical Simulation and Experiments in Microelectronics and Microsystems."

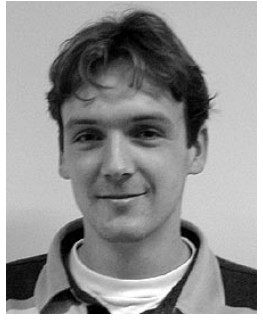

Cornelis van't Hof received the M.S. degree in mechanical engineering from the Delft University of Technology, Delft, The Netherlands, in 1999 where he is currently pursuing the Ph.D. degree in mechanical characterization of curing thermosets.

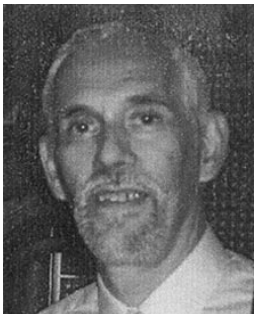

J. F. J. M. Caers received the M.Sc and Ph.D. degrees in applied science from the Catholic University of Leuven, Belgium, in 1972 and 1978, respectively.

He has gained over 25 years of experience in applied material science as a Member of Staff, Catholic University of Leuven (1972-1977), and in several positions in process development within Philips. He has authored and co-authored several papers on these subjects and holds five patent applications. He is now responsible for the competence development of reliability of interconnections of level 2 (substrate level) within Philips Centre for Industrial Technology (CFT). He is currently based in CFT Asia Pacific in Singapore. His major objectives are to integrate quality and reliability right from the start of new process developments and to shorten the reliability qualification time.

Dr. Caers is a member of the Quality and Reliability Chapter, Program Committee, IEEE Electronic Components and Technology Conference (ECTC), and is Evaluator for European consortium projects. 


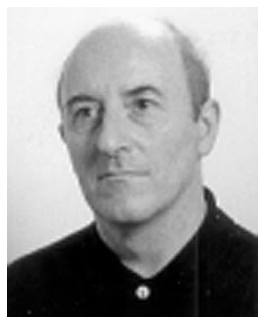

H. J. L. Bressers received the M.S. degree in chemistry/nuclear energy from the State Institute For Nuclear Energy, Mol, Belgium, in 1969.

He joined Philips Research Labs, Eindhoven, The Netherlands, in 1969, gained over 25 years of experience in polymers and process development, and authored or co-authored over 25 scientific publications. $\mathrm{He}$ is currently with Philips Semiconductors as a Senior Scientist focussing on material policy for microelectronics and virtual prototyping of microelectronic packages.

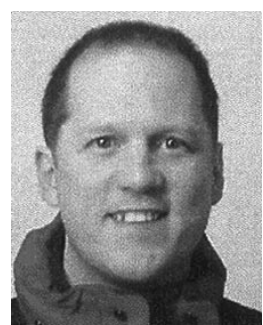

J. H. J. Janssen received the M.S. degree in mechanical engineering from Eindhoven Technical University, Eindhoven, The Netherlands.

He joined Philips CFT, Eindhoven, in 1990 and worked on modeling of dynamics of ultrasonic welding, contamination control in production areas and injection moulding. He moved to Philips Semiconductors in 1996 where he currently is managing the thermal and thermo-mechanical package characterization. He was Philips Semiconductors Manager for the European IST-SEED project and IST-PROFIT and is their voting member of the JEDEC JC-15 committee. 